\title{
Enhancing Perceptual Learning by Combining Practice with Periods of Additional Sensory Stimulation
}

\author{
Beverly A. Wright, ${ }^{1,2}$ Andrew T. Sabin, ${ }^{1}$ Yuxuan Zhang, ${ }^{1}$ Nicole Marrone, ${ }^{1}$ and Matthew B. Fitzgerald ${ }^{3}$ \\ ${ }^{1}$ Department of Communication Sciences and Disorders, and ${ }^{2}$ Northwestern University Institute for Neuroscience, Northwestern University, Evanston, \\ Illinois 60208-3550, and ${ }^{3}$ Department of Otolaryngology, New York University Medical Center, New York, New York 10016
}

Perceptual skills can be improved even in adulthood, but this learning seldom occurs by stimulus exposure alone. Instead, it requires considerable practice performing a perceptual task with relevant stimuli. It is thought that task performance permits the stimuli to drive learning. A corresponding assumption is that the same stimuli do not contribute to improvement when encountered separately from relevant task performance because of the absence of this permissive signal. However, these ideas are based on only two types of studies, in which the task was either always performed or not performed at all. Here we demonstrate enhanced perceptual learning on an auditory frequency-discrimination task in human listeners when practice on that target task was combined with additional stimulation. Learning was enhanced regardless of whether the periods of additional stimulation were interleaved with or provided exclusively before or after target-task performance, and even though that stimulation occurred during the performance of an irrelevant (auditory or written) task. The additional exposures were only beneficial when they shared the same frequency with, though they did not need to be identical to, those used during target-task performance. Their effectiveness also was diminished when they were presented 15 min after practice on the target task and was eliminated when that separation was increased to $4 \mathrm{~h}$. These data show that exposure to an acoustic stimulus can facilitate learning when encountered outside of the time of practice on a perceptual task. By properly using additional stimulation one may markedly improve the efficiency of perceptual training regimens.

\section{Introduction}

Perceptual skills can be improved even in adulthood. This capacity enables optimization of normal abilities and opens treatment options for perceptual disorders. However, a major constraint on the practical application of perceptual learning is that it takes considerable effort. This is because such learning typically does not result from sensory stimulation alone, but rather requires substantial practice performing a perceptual task with relevant stimuli. The need for active practice has led to the idea that task performance provides a permissive signal that allows the accompanying stimulation to drive learning. The lack of learning from stimulus exposures alone, in turn, has been attributed to the absence of this permissive signal. Implicit in these ideas is the assumption that task performance is necessary throughout the entire course of training and thus that only stimulus exposures that occur simultaneously with active practice contribute to learning. We challenge that assumption here by showing that task performance is required for only a portion of each training session. In doing so, we demonstrate that the influences of task performance and additional stimulation can interact temporally

Received Jan. 28, 2010; revised July 27, 2010; accepted Aug. 9, 2010.

This work was supported by the National Institute on Deafness and Other Communication Disorders-National Institutes of Health. We thank Julia Huyck for helpful comments on the manuscript. Donna Bridge, Preethi Chintamaneni, Daniella Karidi, Christine Williams, and Roselyn Wilson helped with data collection.

Correspondence should be addressed to Beverly A. Wright, Department of Communication Sciences and Disorders, and Northwestern University Institute for Neuroscience, 2240 Campus Drive, Northwestern University, Evanston, IL 60208-3550. E-mail: b-wright@northwestern.edu.

DOI:10.1523/JNEUROSCI.0487-10.2010

Copyright $\odot 2010$ the authors $\quad$ 0270-6474/10/3012868-10\$15.00/0 to enhance improvements, providing a means to markedly reduce the effort required for perceptual learning.

The idea that task performance provides a permissive signal that is necessary for learning on many perceptual skills arises primarily from two lines of evidence. First, learning resulting from performing a given target task (the task to be learned) typically does not lead to better behavioral performance on a different, untrained, task even with the same standard stimulus (Karni and Sagi, 1991; Shiu and Pashler, 1992; Treisman et al., 1992; Ahissar and Hochstein, 1993; Levi and Polat, 1996; Crist et al., 1997; Fahle, 1997; Sigman and Gilbert, 2000; Meinhardt, 2002; Meinhardt and Grabbe, 2002). If improvements were driven solely by stimulus exposures, learning should transfer across tasks. Second, physiological changes that have been observed to accompany perceptual learning either do not occur or are markedly reduced when the stimulus exposures are not linked with active performance of a task (Ahissar et al., 1992; Recanzone et al., 1992, 1993; Gao and Suga, 1998; Crist et al., 2001; Bao et al., 2004; Li et al., 2004; Stefan et al., 2004; Blake et al., 2006; Polley et al., 2006).

While the preceding cases clearly demonstrate the need for target-task performance, all of those data were obtained using training regimens in which the task was either always performed or not performed at all. Thus, it is not known whether that active practice must continue throughout each training session or could instead occur only during a portion of each session. To investigate this question, we examined how multiple-session training affected the performance of normal-hearing adults on a target auditory frequency-discrimination task using different training 
A Frequency Discrimination

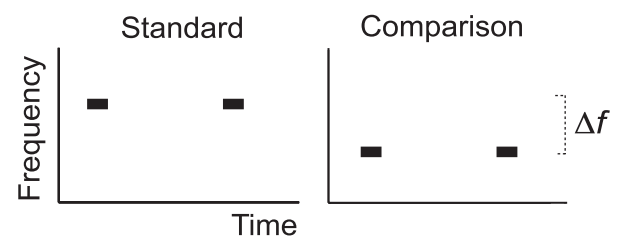

B

Temporal-Interval Discrimination

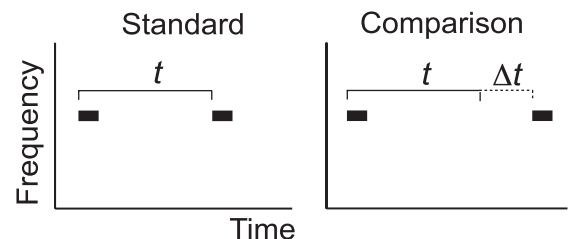

Figure 1. The frequency- and temporal-interval discrimination tasks. Two brief tones were presented in each observation period of a two-presentation, forced-choice trial. In the frequency-discrimination task $(\boldsymbol{A})$, the two tones had a standard frequency $(f)$ in one presentation and a lower comparison frequency in the other $(f-\Delta f)$. In the interval-discrimination task $(\boldsymbol{B})$, the two tones were separated by a standard interval $(t)$ in one presentation and by a longer comparison interval in the other $(t+\Delta t)$. The listener selected the comparison sound (lower frequency or longer interval). In most cases, during training the standard stimulus for both tasks had a frequency of $1 \mathrm{kHz}$ and a temporal interval of $100 \mathrm{~ms}$.

regimens in which practice on this task was or was not paired with extra stimulus exposures. If only stimuli that are presented simultaneously with target-task performance contribute to learning, the additional exposures should provide no benefit. Instead, learning was enhanced in a variety of regimens that provided the additional exposures, indicating that stimulation that occurs outside of the time of task performance can facilitate learning.

\section{Materials and Methods}

\section{General protocol}

Each experiment consisted of an initial familiarization session, a pretraining test, 6-11 training sessions, and a post-training test, all of which were conducted on separate days. During the $\sim 1$ h familiarization session, all listeners completed tone-detection tests in quiet and in noise to introduce them to the laboratory setting and basic testing procedures and to confirm that they had normal hearing. All listeners then completed a $\sim 2 \mathrm{~h}$ pretraining test in which they performed a variety of related tonediscrimination conditions (described below). Subsets of listeners, referred to as the trained listeners, next completed one of 13 different training regimens consisting of $6-11 \sim 1$ h daily sessions during a training phase (described below). The remaining listeners, referred to as controls, received no training. Finally, all listeners completed a post-training test that was identical to the pretraining test. The pre- and post-training tests were separated by $14.6 \mathrm{~d}$ for the trained listeners $(n=98)$ and $12.1 \mathrm{~d}$ for the controls $(n=10)$.

\section{Tasks}

We used two different auditory discrimination tasks in this investigation: frequency discrimination and temporal-interval discrimination. For both tasks, we presented two brief tones in each observation period of a two-presentation, forced-choice trial. In the frequency-discrimination task (Fig. $1 A$ ), the two tones were separated by the same fixed interval $(t)$ in both presentations, but had a standard frequency $(f)$ in one presentation and a lower comparison frequency in the other $(f-\Delta f)$. In the interval-discrimination task (Fig. $1 B$ ), the two tones had the same fixed frequency $(f)$ in both presentations, but were separated by a standard interval $(t)$ in one presentation and by a longer comparison interval in the other $(t+\Delta t)$. Each presentation was marked on a computer screen by a visual display. The onsets of the first tones in the first and second presen- tations were separated by $900 \mathrm{~ms}$. The listener pressed a key on a computer keyboard to indicate which of the two randomly selected presentations contained the comparison sound (lower frequency or longer interval). A visual display indicated whether the response was correct or incorrect after every trial throughout the entire experiment.

\section{Procedure}

We adaptively varied the value of the comparison interval in each 60-trial block to determine the discrimination threshold. The $\Delta f$ or $\Delta t$ changed between trials according to a three-down/one-up rule to determine the value each listener needed to discriminate the comparison stimulus from the standard one on $79.4 \%$ of trials (Levitt, 1971). This value is referred to as the threshold. The initial comparison frequency or interval was always equal to that of the standard, forcing the listener to guess on the first trial. For the frequency-discrimination task, the step size was $0.05 \%$ of the standard frequency until the third reversal, and was $0.01 \%$ thereafter. For the interval-discrimination task, the step size was $10 \%$ of the standard interval until the third reversal, and was $1 \%$ thereafter.

\section{Stimuli}

All sounds were generated digitally and presented to the left ear through headphones. Each 86-dB SPL tone had a total duration of $15 \mathrm{~ms}$, including $5 \mathrm{~ms}$ raised cosine ramps, and was always presented in zero phase. The temporal interval between the two tones in each tone pair was measured from the onset of the first tone to the onset of the second tone.

\section{Pretraining and post-training tests}

In the pre- and post-training tests, all listeners completed five threshold estimates (300 trials) in three frequency-discrimination conditions and three temporal-interval discrimination conditions, with the following exception. In place of the interval conditions, listeners in one group [All-Freq (360 trials), see below] instead completed one temporalinterval discrimination condition and two additional frequencydiscrimination conditions. The condition order was randomized across listeners, but fixed between the pre- and post-training tests. Because learning on frequency discrimination was the focus of this investigation, we only report the results for the three frequency-discrimination conditions completed by all listeners here. One of these conditions was the target (standard: $1 \mathrm{kHz}, 100 \mathrm{~ms}$ ). This was the condition listeners practiced during periods of target-task performance. The other two, untrained, conditions differed from the trained one either in the temporal interval (standard: $1 \mathrm{kHz}, 50 \mathrm{~ms}$ ) or the frequency (standard: $4 \mathrm{kHz}, 100$ $\mathrm{ms}$ ) of the standard.

\section{Training regimens}

Initial experiments. In the initial experiments, the trained listeners participated in one of five different $6-11 \mathrm{~d}$ training regimens (Fig. $2 A$ ). In the three key regimens, listeners practiced the target task (frequency discrimination with a $1 \mathrm{kHz}, 100 \mathrm{~ms}$ standard) 360 trials per day for 6-8 d. These daily trials were presented in three sets of 120 trials each and alternated with one of three additional experiences, of equal duration, depending on the regimen. In one regimen, listeners performed a written symbol-to-number matching task in silence (Freq-alternating-with-Silence; $n=7)$. In another, they performed that same written matching task while stimuli were played in the background (Freq-alternating-withSound; $n=8$ ). In the symbol-to-number matching task, listeners wrote on a worksheet the number corresponding to each of a series of printed images presented in random order, based upon a key in which each number was associated with a different image. The background sounds consisted of presentations of stimuli from representative adaptive tracks from a temporal-interval discrimination task that used the same standard as the target frequency-discrimination task (120 "trials" per set, 360 total per session). Note that these background sounds differed in temporal interval across presentations, but all had the same frequency. In the third regimen, listeners actually performed that temporal-interval discrimination task (Freq-alternating-with-Interval; $n=8$ ). In the two remaining regimens, used for comparison, listeners practiced either only the temporal-interval discrimination task (All-Interval (900 trials); $n=$ 6 ) or only the target frequency-discrimination task (All-Freq (900 trials); $n=8) 900$ trials per day for $10-11 \mathrm{~d}$. 
A

\section{Daily Training Regimens (practiced 6-11 days)}

Freq-alternating-with-Silence: 7 days

Freq Silence $\square$

Freq-alternating-with-Sound: 7 days

Freq ESound

Freq-alternating-with-Interval: 6-8 days

Freq Interval
All-Interval (900 trials): $10-11$ days
\begin{tabular}{|l|l|l|l|l|l|l|l|}
\hline Interval & & & & & & & (or equivalent) \\
\hline
\end{tabular}

All-Freq (900 trials): 10-11 days

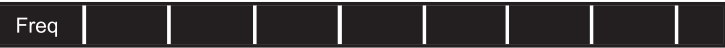

B
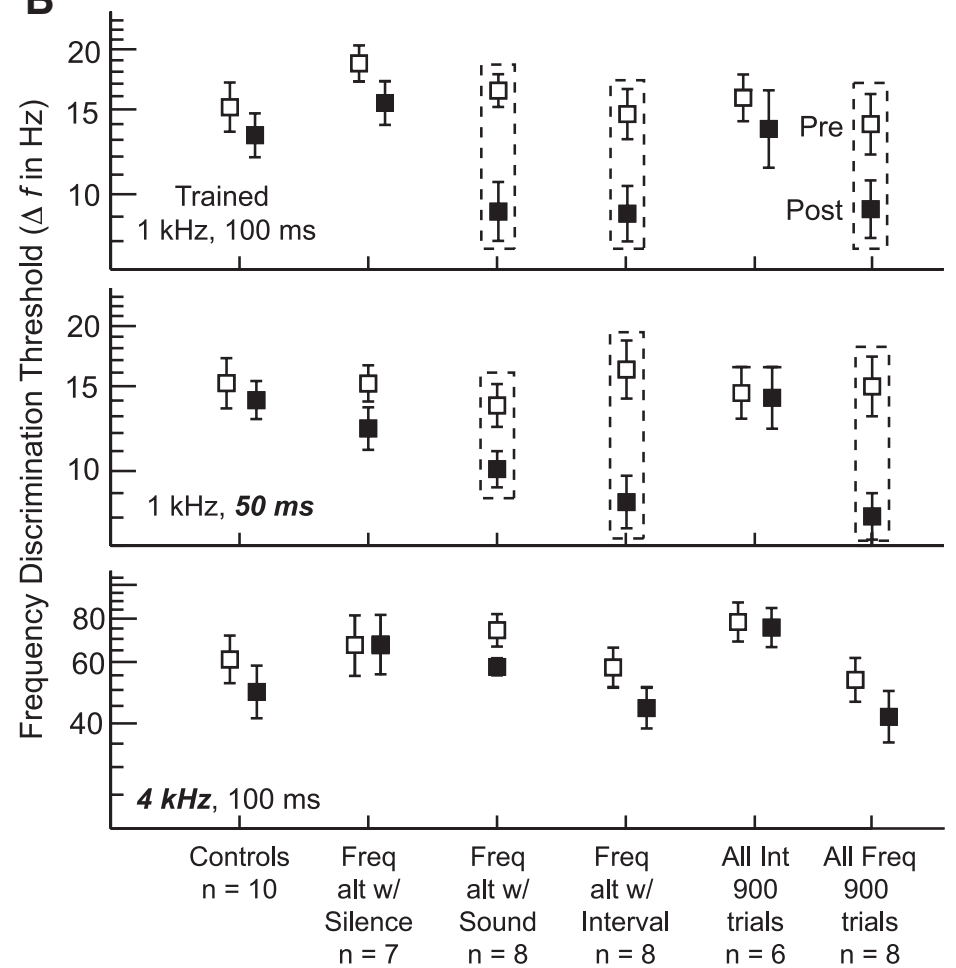

\section{C}
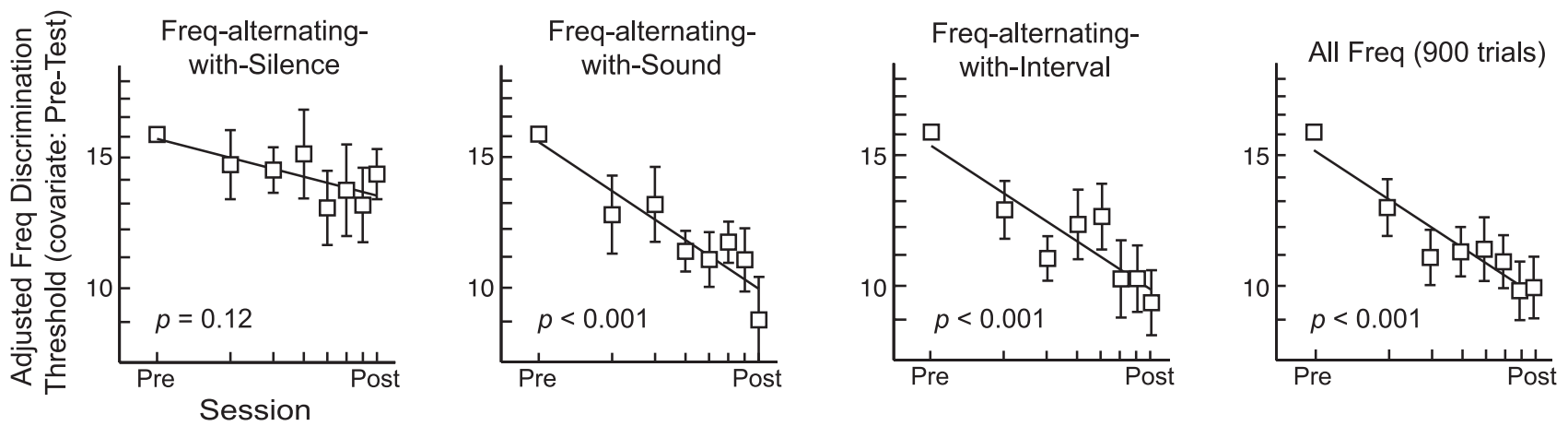

Figure 2. Initial experiments. A, Training regimens. Different groups of adults with normal hearing were given one of five daily training regimens for $6-11 \mathrm{~d}$. In the three key regimens, in each session, practice on a target frequency-discrimination task (standard: $1 \mathrm{kHz}, 100 \mathrm{~ms}$ ) alternated with performance of either (1) a written symbol-to-number matching task in silence (Freqalternating-with-Silence), (2) the written task while stimuli were played in the background (Freq-alternating-with-Sound), or (3) a temporal-interval discrimination task (Freq-alternating-withInterval). The additional stimulus exposures (background sounds or stimuli for temporal-interval discrimination) had the same standard as in the target frequency-discrimination task, but varied in temporal interval rather than frequency. In the two remaining regimens all of the practice was on either the temporal-interval discrimination task [All-Interval (900 trials)] or the target frequency-discrimination task [All-Freq ( 900 trials)], again with the $1 \mathrm{kHz}, 100 \mathrm{~ms}$ standard. Each box represents 120 trials or the equivalent. $\boldsymbol{B}$, Pre- and post-training data. Mean frequencydiscrimination thresholds ( $\Delta$ in $\mathrm{Hz}$ for $79.4 \%$ correct) before (open squares) and after (filled squares) completing one of the five multiple-day training regimens ( $n=6-8$ per trained group) or receiving no training over that same time period (controls; $n=10$ ). Results are shown for the standard stimulus used in all of the training regimens (top: $1 \mathrm{kHz}, 100 \mathrm{~ms}$ ) and for the two untrained standard stimuli used to test the generalization of learning (middle: $1 \mathrm{kHz}, 50 \mathrm{~ms}$ and bottom: $4 \mathrm{kHz}, 100 \mathrm{~ms}$ ). Error bars indicate \pm SEM. Dashed boxes indicate significantly greater improvement between the pre- and post-training tests by a trained group than controls $(p<0.05)$. C, Performance across sessions. Group mean thresholds (squares) across sessions for the target frequencydiscrimination task (standard: $1 \mathrm{kHz}, 100 \mathrm{~ms}$ ) for each of the four groups who practiced frequency discrimination (panels), with regression lines fitted to the thresholds on the log of the session number. Error bars indicate \pm SEM. Thresholds were adjusted to account for individual differences in pretraining threshold (Cohen, 1988). The $p$-values reflect whether the slope of the regression line was significantly different from zero. The key result is that the combination of practice on the target frequency-discrimination task and additional stimulus exposures (Freq-alternating-withSound and Freq-alternating-with-Interval) facilitated learning on frequency discrimination. Learning was enhanced relative to when the training involved only the target-task performance portion of the combined regimens (Freq-alternating-with-Silence), as well as to when stimulus exposures in greater numbers than in those regimens were presented in the absence of target-task performance [All-Interval ( 900 trials)].

Follow-up experiments. In follow-up experiments, eight new groups of listeners each participated in a different training regimen. These regimens differed from each other in terms of the presentation order of, the duration between, and the stimuli used during the periods of target-task performance and additional stimulus exposure. In all eight new regimens, listeners again practiced the target task (frequency discrimination with a $1 \mathrm{kHz}, 100 \mathrm{~ms}$ standard) for 360 trials per day for $6-7 \mathrm{~d}$, but these trials occurred consecutively rather than in separate 120 -trial sets as in the alternating regimens of the initial experiments. In one regimen, the listeners completed only these frequency-discrimination trials (All-Freq (360 trials); $n=8$ ) (Fig. 3A). In the other seven regimens, in addition to the frequency-discrimination trials, the listeners also performed 360 consecutive trials per day of temporal-interval discrimination or performed the written symbol-to-number matching task while 360 "trials" 
A

$$
\begin{aligned}
& \text { Daily Training Regimens } \\
& \text { (practiced 6-7 days) }
\end{aligned}
$$

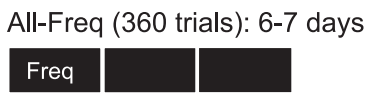

120 trials (or equivalent)

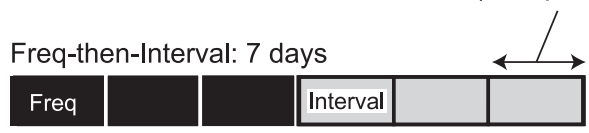

Interval-then-Freq: 7 days

\begin{tabular}{|l|l|l|l|l|}
\hline Interval & & & Freq & \\
\end{tabular}

Sound-then-Freq: 7 days

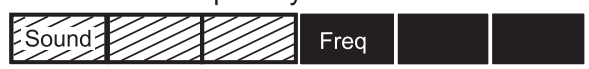

B

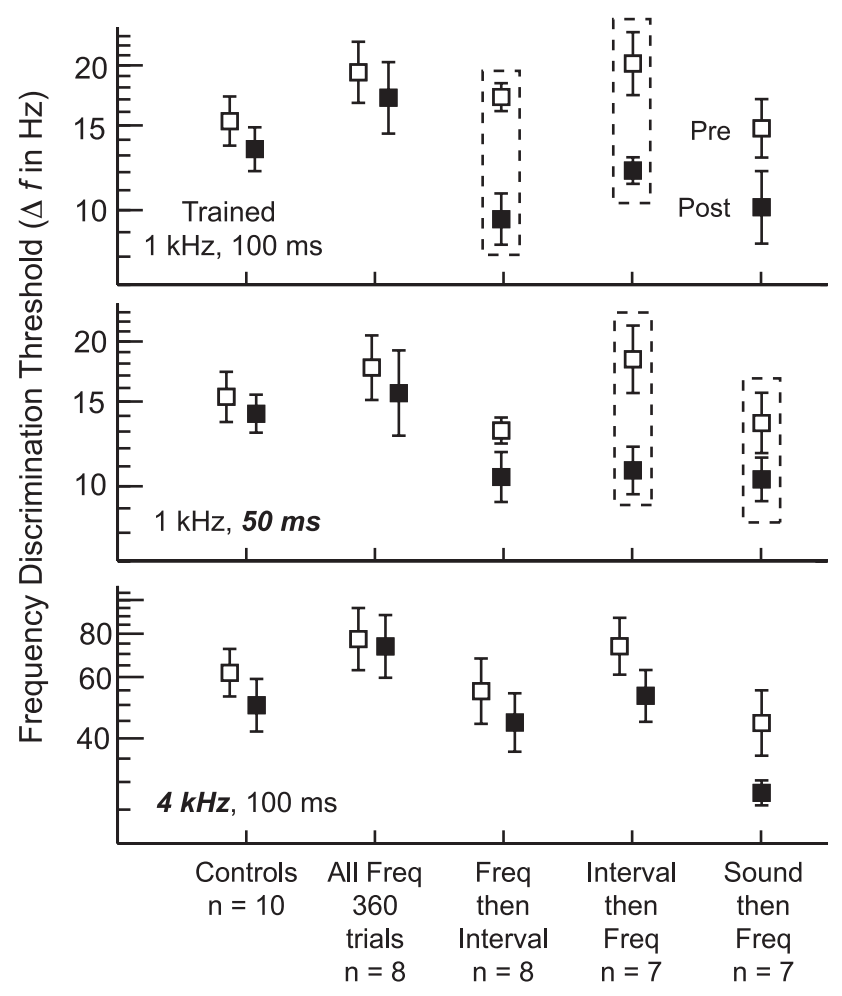

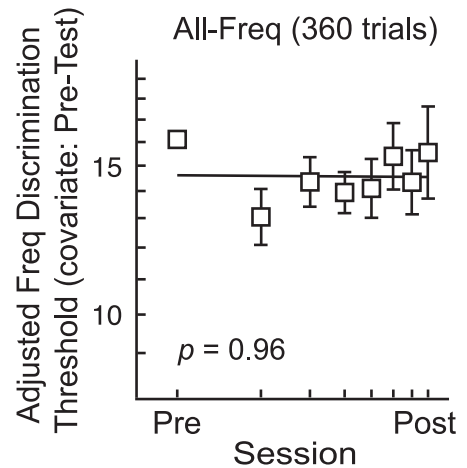
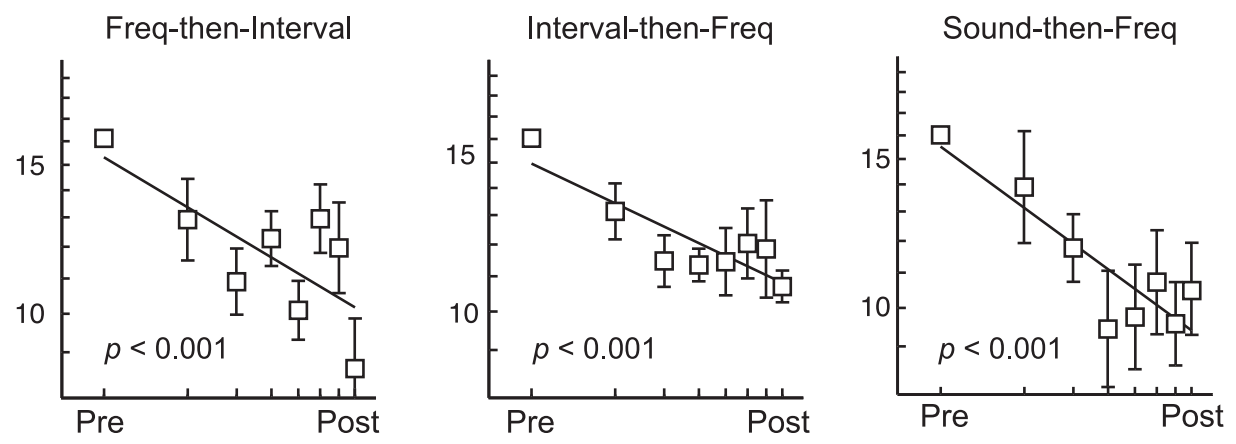

Figure 3. Follow-up experiments: presentation order. A, Training regimens. In one regimen all of the practice in each session was on a target frequency-discrimination task (standard: $1 \mathrm{kHz}, 100 \mathrm{~ms}$ ) [All-Freq (360 trials)]. In the other three regimens, in each session, practice on the target frequency-discrimination task occurred either (1) exclusively before performance of a temporal-interval discrimination task (Freq-then-Interval), (2) exclusively after performance of that temporal-interval task (Interval-then-Freq), or (3) exclusively after performance of a written symbol-to-number matching task while stimuli were played in the background (Sound-then-Freq). Otherwise, as in Figure 2. B, Pre- and post-training data. As in Figure 2, with the data from controls (Fig. 2) replotted for comparison. C, Performance across sessions. As in Figure 2. The key result here is that the additional stimulus exposures enhanced learning on the target frequency-discrimination task regardless of whether those exposures were presented exclusively before or after target-task performance.

of the same background sounds as in the initial experiments were presented. Of these regimens, in one, the listeners practiced the target frequency-discrimination task immediately before performing the temporal-interval discrimination task (Freq-then-Interval; $n=8$ ) (Fig. $3 A$ ). In two other regimens they practiced the target task immediately after, rather than before, performing the temporal-interval discrimination task (Interval-then-Freq; $n=7$ ) or performing the symbol-tonumber matching task in the presence of the background sounds (Sound-then-Freq; $n=7$ ) (Fig. $3 A$ ). In another two regimens the listeners completed the temporal-interval task either 15 min (Freq-15 minInterval; $n=9$ ) or $4 \mathrm{~h}$ (Freq-4 h-Interval; $n=8$ ) after practicing the target frequency-discrimination task (Fig. $4 A$ ). In all of these regimens the standard sound was the same for both tasks $(100 \mathrm{~ms}, 1 \mathrm{kHz})$. In the remaining two regimens listeners practiced frequency discrimination followed by temporal-interval discrimination, but the standard stimuli differed between the two tasks. The temporal-interval task used a standard that differed from that in the target frequency-discrimination task
$(1 \mathrm{kHz}, 100 \mathrm{~ms})$ only in frequency in one regimen $(4 \mathrm{kHz}, 100 \mathrm{~ms}$; Freq-then-Interval (different frequencies); $n=7$ ) and only in temporal interval in the other $(1 \mathrm{kHz}, 350 \mathrm{~ms}$; Freq-then-Interval (different intervals); $n=7$ ) (Fig. $5 A$ ).

\section{Listeners}

One hundred and eight listeners ( 69 females) ranging in age from 18 to 30 years [mean 20.7 years (SD 3.2)] were paid for their participation. All had normal hearing and no previous experience with psychoacoustic tasks. Some data from the All-Freq (360 trials), All-Freq (900 trials) and AllInterval (900 trials) groups were reported previously (Wright and Sabin, 2007), but the post-training performance of these groups is shown for the first time here.

\section{Analyses}

All analyses were conducted on log-transformed $\left(\log _{10} \mathrm{~Hz}\right)$ thresholds. The data of individuals whose pretraining thresholds were $>2$ SDs above 
A

\section{Daily Training Regimens (practiced 6-7 days)}

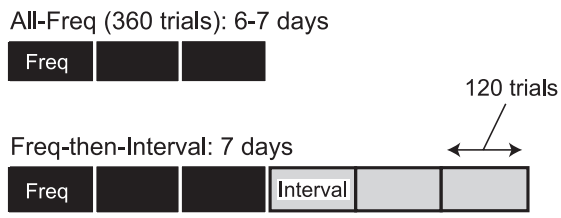

Freq-15 min-Interval: 7 days

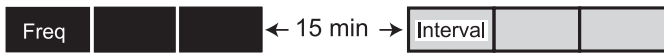

Freq-4 hr-Interval: 7 days

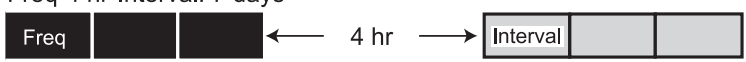

C
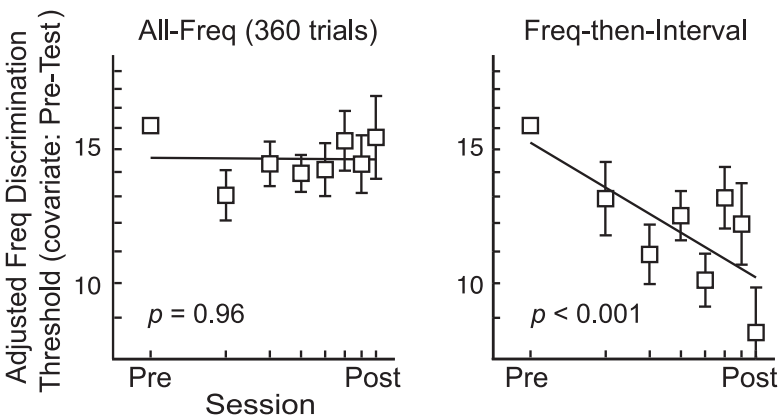

B

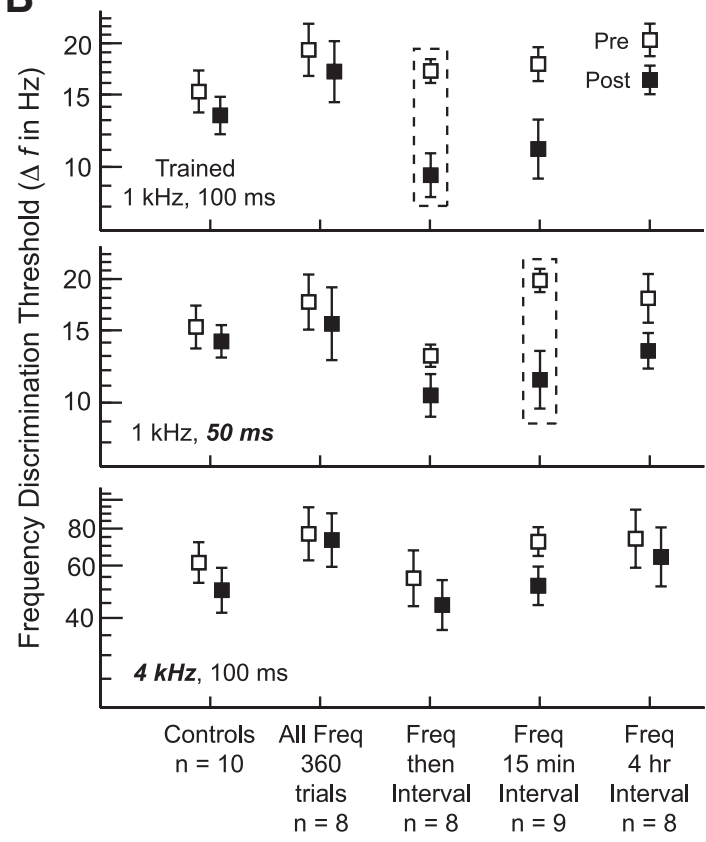

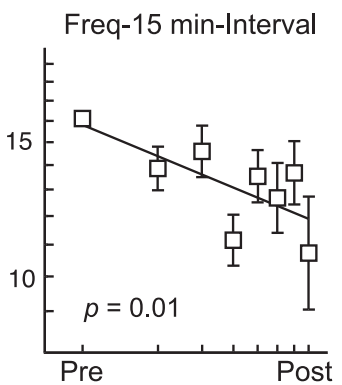

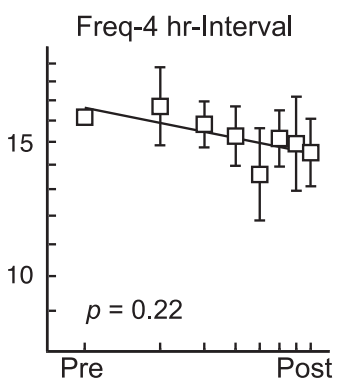

Figure 4. Follow-up experiments: temporal separation. $A$, Training regimens. In one regimen all of the practice in each session was on a target frequency-discrimination task (standard: $1 \mathrm{kHz}$, 100 ms) [All-Freq (360 trials)]. In the other three regimens, in each session, practice on the target frequency-discrimination task occurred before performance of a temporal-interval discrimination task using the same standard stimulus, with the two events separated by either (1) 0 min (Freq-then-Interval), (2) $15 \mathrm{~min}$ (Freq-15 min-Interval), or (3) $4 \mathrm{~h}$ (Freq-4 h-Interval). 0therwise, as in Figure 2. $\boldsymbol{B}, \boldsymbol{C}$, Pre- and post-training data $(\boldsymbol{B})$ and performance across sessions ( $\boldsymbol{C}$. As in Figure 2, with the data from controls (Fig. 2) and from the All-Freq ( 360 trials) and Freq-then-Interval groups (Fig. 3) replotted for comparison. The key result here is that some benefit from the additional stimulus exposures remained when they were presented 15 min, but not $4 \mathrm{~h}$, after performance of the target frequency-discrimination task.

the mean of all 108 listeners were removed from the analyses on a condition-by-condition basis (5.4\% of the total values). The pretraining thresholds combined across all groups were positively correlated with the post-training thresholds for each of the three different standard stimuli: $1 \mathrm{kHz}, 100 \mathrm{~ms}(r=0.61, p<0.0001), 1 \mathrm{kHz}, 50 \mathrm{~ms}(r=0.53, p<0.0001)$, and $4 \mathrm{kHz}, 100 \mathrm{~ms}(r=0.78, p<0.0001)$. Therefore to evaluate the influence of training, we used analyses of covariance (ANCOVAs) to adjust the post-training thresholds of each group, using pretraining thresholds as the covariate. The main statistical conclusions are based on separate ANCOVAs comparing the post-training thresholds of each trained group for each standard to those of the controls. In the few cases in which the heterogeneity of regression was significant, we instead evaluated whether there was a significant interaction in a two group (trained vs control) by two time (pre- vs post-training test) ANOVA. These analyses followed omnibus ANCOVAs for each standard: $1 \mathrm{kHz}, 100 \mathrm{~ms}$ $\left(F_{(1,88)}=2.56, p=0.005\right), 1 \mathrm{kHz}, 50 \mathrm{~ms}\left(F_{(1,86)}=3.36, p<0.0001\right)$, and $4 \mathrm{kHz}, 100 \mathrm{~ms}\left(F_{(1,86)}=1.51, p=0.15\right)$. The test for heterogeneity of regression was not significant for any of these analyses: $1 \mathrm{kHz}, 100 \mathrm{~ms}$ $\left(F_{(13,75)}=0.84, p=0.61\right), 1 \mathrm{kHz}, 50 \mathrm{~ms}\left(F_{(13,73)}=0.65, p=0.80\right)$, and $4 \mathrm{kHz}, 100 \mathrm{~ms}\left(F_{(13,73)}=0.98, p=0.48\right)$. We also fitted separate regression lines through the daily mean frequency-discrimination thresholds of the individual listeners against the log of the session number for each of the groups who received training on frequency discrimination. These fits included data from the pretraining test, the first six training sessions (the maximum number obtained from every listener), and the posttraining test. The training-phase and post-training thresholds were adjusted using pretraining threshold as the covariate (Cohen, 1988).

\section{Results}

\section{Initial experiments}

Of the three key training regimens used in the initial investigation, only the two that provided additional stimulus exposures yielded learning between the pre- and post-training tests (Fig. $2 B)$. The Freq-alternating-with-Silence group, who did not receive the additional exposures, did not improve significantly more than controls either with the trained standard $(1 \mathrm{kHz}, 100$ ms) $\left(F_{(1,14)}=0.001, p=0.97\right)$ or with the standard that had the untrained interval $(1 \mathrm{kHz}, 50 \mathrm{~ms})\left(F_{(1,14)}=1.47, p=0.25\right)$ and actually showed a trend toward less improvement than controls with the standard that had the untrained frequency $(4 \mathrm{kHz}, 100$ $\mathrm{ms})\left(F_{(1,14)}=4.06, p=0.06\right)$. In contrast, the Freq-alternatingwith-Sound and Freq-alternating-with-Interval groups, who 
A

\section{Daily Training Regimens (practiced 6-7 days)}
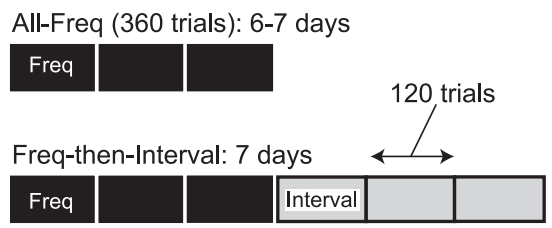

Standard for Interval

$1 \mathrm{kHz}, 100 \mathrm{~ms}$

Freq-then-Interval (different frequencies): 7 days

\begin{tabular}{|c|c|c|}
\hline & 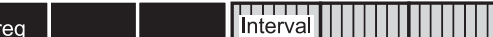 \\
\hline
\end{tabular}

Freq-then-Interval (different intervals): 7 days

\begin{tabular}{l|l|l|l|l|}
\hline Freq & & Interval \\
\hline
\end{tabular}

$1 \mathrm{kHz}, 350$ ms

$\underline{\text { Standard for Freq }}$

(in all cases)

$1 \mathrm{kHz}, 100 \mathrm{~ms}$
B

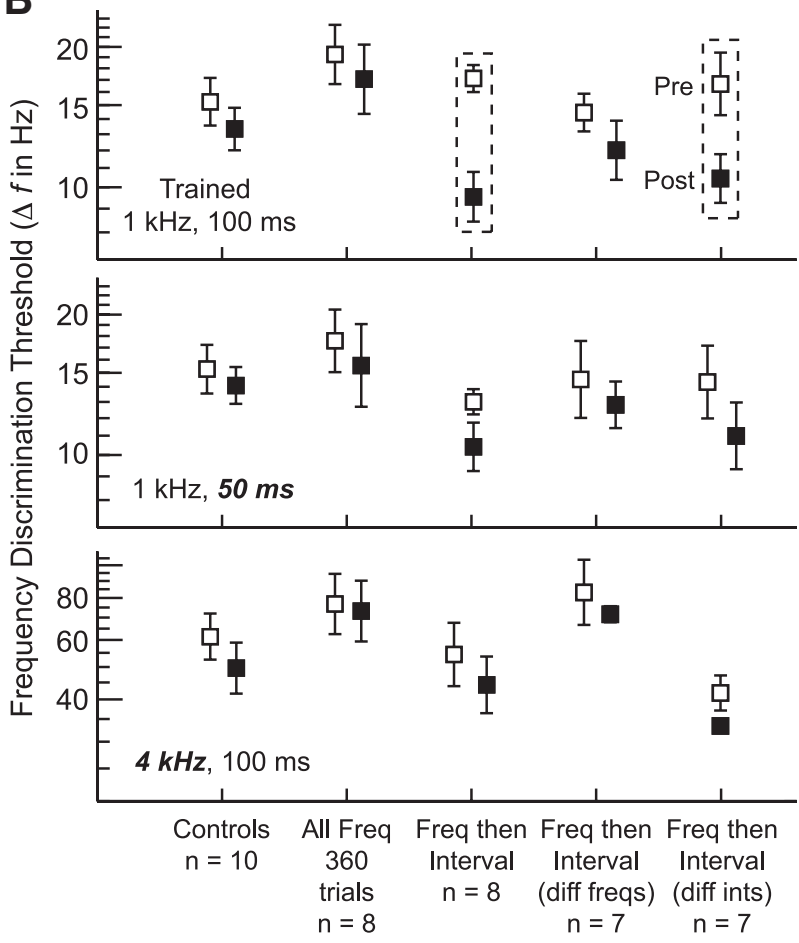

C

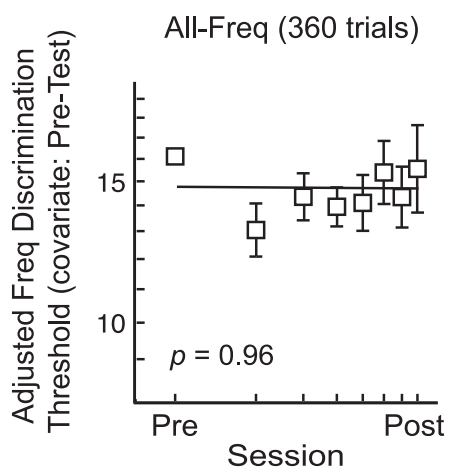

Freq-then-Interval

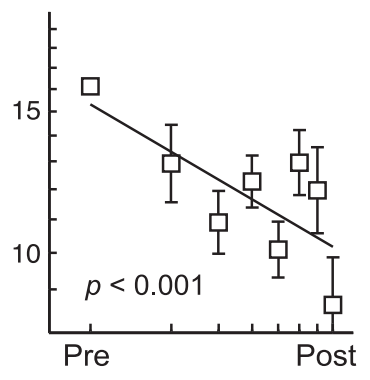

Freq-then-Interval (different frequencies)

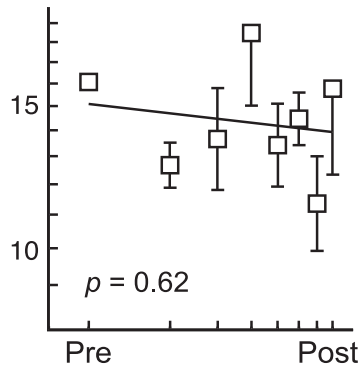

Freq-then-Interval (different intervals)

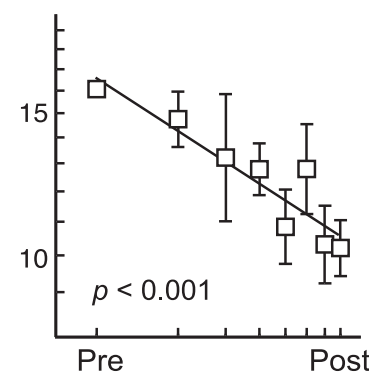

Figure 5. Follow-up experiments: stimulus dependence. $\boldsymbol{A}$, Training regimens. In one regimen all of the practice in each session was on a target frequency-discrimination task (standard: $1 \mathrm{kHz}$, $100 \mathrm{~ms}$ ) [All-Freq (360 trials)]. In the other three regimens, in each session, practice on the target frequency-discrimination task immediately preceded performance of a temporal-interval discrimination task. As compared with the standard stimulus used in the target frequency-discrimination task, the standard for the temporal-interval discrimination task either (1) was the same (1 kHz, 100 ms; Freq-then-Interval), (2) had a different frequency but the same temporal interval [4 kHz, $100 \mathrm{~ms}$; Freq-then-Interval (different frequencies)], or (3) had the same frequency but a different temporal interval [1 kHz, $50 \mathrm{~ms}$; Freq-then-Interval (different intervals)]. Otherwise, as in Figure 2. B, $\boldsymbol{C}$, Pre- and post-training data (B) and Performance across sessions ( $\boldsymbol{C}$. As in Figure 2, with the data from controls (Fig. 2) and from the All-Freq (360 trials) and Freq-then-Interval groups (Fig. 3) replotted for comparison. The key result here is that the additional stimulus exposures led to enhanced learning on the target frequency-discrimination task only when those exposures had the same frequency as, though they did not have to be identical to, the stimuli encountered during performance of the target task.

both received the additional exposures, learned significantly more than controls with the trained standard $\left(F_{(1,15)}=8.38, p=\right.$ $0.01 ; F_{(1,15)}=9.11, p=0.009$, respectively) and generalized their learning to the standard with the untrained interval $\left(F_{(1,15)}=\right.$ $\left.6.90, p=0.02 ; F_{(1,15)}=20.00, p=0.0004\right)$, though not to the one with the untrained frequency $\left(F_{(1,15)}=0.002, p=0.96 ; F_{(1,15)}=\right.$ $0.21, p=0.65)$.

The learning afforded by the additional stimulus exposures did not result simply from exposure to the trained standard, to stimuli with different temporal intervals or from the transfer of learning from temporal-interval to frequency discrimination (Fig. 2B). The All-Interval (900 trials) group was exposed to stimuli with different temporal intervals just like the Freq-alternating-with-Sound and Freq-alternating-with-Interval groups and even received more total exposures to the standard used in the frequency-discrimination training than did those additional-exposure groups (900 vs 720 exposures per day; $10 \mathrm{vs} 7 \mathrm{~d}$ ). Nevertheless, the All-Interval (900 trials) listeners showed no more learning than controls on any of the three frequency-discrimination conditions (trained standard: $F_{(1,13)}=$ $0.007, p=0.93$; untrained interval: $F_{(1,13)}=0.07, p=0.80$; untrained frequency: $\left.F_{(1,12)}=1.89, p=0.19\right)$.

It is noteworthy that the two regimens that combined targettask performance and additional stimulus exposures yielded the same improvement pattern as did extended training on the target task throughout each training session (Fig. 2B). The All-Freq (900 trials) group improved significantly more than controls with the trained standard $\left(F_{(1,15)}=6.89, p=0.02\right)$ and untrained interval $\left(F_{(1,15)}=20.50, p=0.0004\right)$ but not with the untrained 
frequency $\left(F_{(1,15)}=0.07, p=0.79\right)$ just as the two additionalexposure groups had. Thus, the combination of 360 daily trials of target-task performance and 360 daily "trials" of additional stimulus exposures had the same effect as a regimen that provided an even greater number of stimulus exposures, all of which were encountered while performing the target task.

The benefit received from the additional stimulus exposures was also evident over the course of training (Fig. 2C). For the Freq-alternating-with-Silence group, the slope of the regression line fitted to the frequency-discrimination thresholds against the $\log$ of the session number was negative, but was not statistically significant, indicating minimal improvement at best (slope $=$ $-0.08, p=0.12$ ). However, the slope was significant for the Freq-alternating-with-Sound (slope $=-0.22, p<0.0001$ ) and Freq-alternating-with-Interval (slope $=-0.21, p=0.0001$ ) groups, indicating systematic and gradual learning with these additional-exposure regimens. The slope was also significant for the All-Freq (900 trials) group (

\section{Follow-up experiments}

\section{Presentation order}

The additional stimulus exposures yielded enhanced learning on the target task regardless of whether they were presented exclusively before or after target-task performance (Fig. $3 B, C$ ). The All-Freq (360 trials) group, who practiced all of the trials of the target frequency-discrimination task consecutively and did not receive additional exposures, showed no improvement over the course of training (slope $=-0.002, \mathrm{p}=0.96$ ) and did not improve significantly more than controls between the pre- and posttraining tests on any of the three standards (trained standard: $F_{(1,15)}=0.22, p=0.64$; untrained interval: heterogeneity of regression: $F_{(1,13)}=5.85, p=0.03$, ANOVA group $\times$ time: $F_{(1,15)}=$ $0.07, p=0.80$; untrained frequency: $\left.F_{(1,15)}=1.90, p=0.19\right)$. In contrast, both the Freq-then-Interval and Interval-then-Freq groups learned significantly across sessions (slope $=-0.20, p=$ 0.0008 ; slope $=-0.16, p=0.0005$, respectively) and improved significantly more than controls on the trained standard $\left(F_{(1,13)}=\right.$ 8.02, $\left.p=0.01 ; F_{(1,13)}=5.10, p=0.04\right)$. The Sound-then-Freq group also improved significantly over the course of training (slope $=-0.24, p=0.0006)$ and showed a trend toward greater improvement than controls with the trained standard $\left(F_{(1,14)}=\right.$ $3.78, p=0.07)$. However, while learning enhancement on the target task occurred for both presentation orders of the additional exposures and target-task performance, only one order clearly yielded generalization. The two groups who received the additional exposures first in each session (Interval-then-Freq and Sound-then-Freq) improved significantly more than controls with the untrained standard interval $\left(F_{(1,13)}=8.35, p=0.01\right.$; $F_{(1,13)}=4.53, p=0.05$, respectively), though not with the untrained standard frequency $\left(F_{(1,14)}=0.67, p=0.42\right.$; heterogeneity of regression $F_{(1,13)}=9.98, p=0.007$, group $\times$ time ANOVA: $\left.F_{(1,15)}=1.92, p=0.19\right)$. The learning in the group who received the additional exposures second (Freq-then-Interval) instead appeared to be specific to the trained standard (untrained frequency: $F_{(1,15)}=0.001, p=0.97$; untrained interval: $F_{(1,13)}=2.38, p=0.15$; see below for an analysis that addresses the relatively low starting threshold in this group on the untrained interval). Thus, the regimens in which the additional stimulus exposures preceded targettask performance yielded the generalization pattern that was most similar to that obtained with extensive daily training on frequencydiscrimination alone [All-Freq (900 trials)].
Temporal separation

Some benefit from the additional stimulus exposures remained when they were presented $15 \mathrm{~min}$, but not $4 \mathrm{~h}$, after target-task performance (Fig. $4 B, C$ ). The listeners who received the additional exposures $4 \mathrm{~h}$ after practicing the target frequencydiscrimination task each day (Freq-4 h-Interval) did not improve over the course of training ( lope $=-0.06, p=0.22$ ) or distinguish their performance from that of controls on any of the three standards (trained standard: heterogeneity of regression $F_{(1,14)}=$ 5.53, $p=0.03$, group $\times$ time ANOVA $F_{(1,16)}=0.34, p=0.57$; untrained interval: $F_{(1,15)}=0.94, p=0.35$, untrained frequency: $\left.F_{(1,15)}=0.43, p=0.52\right)$. When the separation between the two events was reduced to $15 \mathrm{~min}$ (Freq-15 min-Interval), the listeners did improve significantly across sessions ( slope $=-0.14, p=$ 0.01 ), but did not improve significantly more than controls between the pre- and post-training tests $\left(F_{(1,15)}=2.13, p=0.17\right)$ on the trained standard, suggesting that the learning enhancement had begun to decline over this time period. Curiously this group showed a trend toward greater improvement than controls with the untrained standard interval $\left(F_{(1,15)}=4.09, p=0.06\right)$ in contrast to the apparent lack of generalization to this standard shown by the listeners for whom there was no break between the two events. This pattern raises the possibility that the break may have had different effects on learning and generalization.

\section{Stimulus dependence}

The additional exposures led to enhanced learning only when they shared a key feature with, but did not have to be identical to, those encountered during target-task performance (Fig. $5 B, C$ ). The listeners who received additional exposures that had a different frequency than that of the standard in the target frequencydiscrimination task (Freq-then-Interval) (different frequencies) did not learn across sessions (slope $=-0.04, p=0.62$ ) or improve more than controls between the pre-and post-training tests (trained standard: $F_{(1,13)}=0.26, p=0.62$; untrained interval: $F_{(1,14)}=0.51, p=0.49$; untrained frequency: $F_{(1,9)}=0.04, p=$ $0.85)$. When the additional exposures instead had the same frequency as but a different temporal-interval than the standard of the target-task (Freq-then-Interval) (different intervals), the listeners improved over the course of training ( 0.0003 ) and also improved more than controls between the pre- and post-training tests on the trained standard $\left(F_{(1,14)}=\right.$ $7.56, p=0.02)$. However, they did not appear to generalize this learning to either the untrained interval $\left(F_{(1,13)}=2.23, p=0.16\right)$ or frequency $\left(F_{(1,13)}=0.04, p=0.85\right)$. While this specificity could have been influenced by the different stimuli encountered during the periods of additional exposures and target-task performance, another possibility given the available data is that it resulted from having received the additional exposures immediately after performing the target-task. The Freq-then-Interval group, who received the exact same training but using standard stimuli that did not differ between the two events, also did not seem to generalize their learning. It is worth noting that the lack of improvement on the untrained interval in these two groups does not appear to have arisen simply because these groups had among the lowest pretraining thresholds with that standard. Listeners with the highest pretraining thresholds across these two groups combined $(n=8)$ still did not improve significantly more than controls on the untrained interval $\left(F_{(1,15)}=1.18, p=0.29\right)$, though their mean pretraining threshold on that condition was comparable to that of the other groups $(16.3 \mathrm{~Hz})$. 


\section{Discussion}

The present results show that a variety of combinations of active task performance and periods of additional stimulus exposure enhanced learning on a target auditory frequency-discrimination task. Learning was enhanced relative to when the training involved only the target-task performance portion of the combined regimens, as well as to when stimulus exposures in greater numbers than in those regimens were presented in the absence of target-task performance. This learning enhancement occurred regardless of whether the additional exposures were encountered while performing a non-target auditory task, or were simply presented in the background during the performance of a written task. It also arose both when the periods of additional stimulus exposure alternated with and when they came exclusively immediately before or after those of target-task performance, but not when those periods were separated by $4 \mathrm{~h}$ or involved different stimulus frequencies. The combined regimens generated as much improvement as did practicing the target task throughout the entire training period. Thus, while task performance was necessary for learning on this task, it was only required for a portion of the training. The necessity for some target-task performance distinguishes the current demonstration from incidental learning, in which improvement on a target task occurs solely through performance of a non-target task that employs the same stimuli (Nissen and Bulemer, 1987). It also separates these results from cases in which learning occurs merely from stimulus exposures, in the absence of any task performance with those stimuli at all (Saffran et al., 1996; Godde et al., 2000; Watanabe et al., 2001; Maye et al., 2002). The capacity for stimulus exposures presented outside of the period of target-task performance to contribute to improvement indicates that learning can be enhanced by a temporal interaction between these two quite different modes of experience.

An emergent conception of perceptual learning is that learning occurs when a neural process that limits performance on the trained skill is placed in a sensitized state in which it can be modified and is then stimulated sufficiently while in that state (Seitz and Dinse, 2007; Wright and Zhang, 2009). It is thought that the sensitized state is brought about through permissive signals that are typically and optimally provided by performance of the target task, with attention or reward as candidates (Ahissar and Hochstein, 2004; Seitz and Watanabe, 2005; Gilbert and Sigman, 2007). The necessary stimulation while in this state comes through stimulus exposures (Wright and Sabin, 2007). The present results fit within, but help to expand, this conception. The improvement on frequency discrimination following practice on that task, but not following practice only on temporal-interval discrimination is consistent with the idea that performance of the target task provided the permissive signals needed to sensitize the neural processes to be modified. Likewise, the presence of learning on frequency discrimination when that task was practiced 900 trials per day but not when it was practiced 360 trials per day (see also Wright and Sabin, 2007) supports the idea that there must be sufficient stimulation of the sensitized processes for behaviorally observable learning to arise.

In this framework, the new information here is that stimulation that occurs outside of the time of the direct activation of the sensitizing permissive signals can contribute to learning. The current data lead to this idea because listeners improved more on frequency discrimination when the additional stimulus exposures, which helped provide the sufficient stimulation, and target-task performance, which provided the necessary permissive signals, were combined in either order, than when either element was provided alone. That the effectiveness of the combination of these two elements began to decline within minutes and was gone within hours of their separation suggests that the influence of their pairing is less likely to be a component of the prolonged consolidation phase, during which what has been learned is transferred from a fragile to a more stable state, than of the acquisition phase, during which the experiences leading to learning are obtained (for recent evidence that these two phases are at least partially separable in perceptual learning, see Banai et al., 2010). It also reduces the possibility that the observed enhancement resulted from the addition of the independent influences of these two elements rather than from the presence of one element directly altering the response to the other.

The current data further suggest that the influence of both the direct activation of the permissive signals and of the additional stimulation spread beyond the time periods in which they occurred. It appears that the influence of the permissive signals spread to the subsequent periods of additional stimulation, because learning was enhanced when the target-task performance preceded the presentation of the additional stimulus exposures. This outcome raises the possibility that task performance led subsequent stimulation to have the same effect as if it had actually occurred during the direct activation of the permissive signals. Consistent with this idea, brief inducing events can lead to persistent physiological changes (Näätänen et al., 1993; Fritz et al., 2003; Weinberger, 2004; Clapp et al., 2005; Froemke et al., 2007). For example, alterations in auditory receptive field tuning induced by performance of a well learned task lasted minutes to hours after task-performance ceased (Fritz et al., 2003) and changes in the strengths of synaptic excitation and inhibition in auditory cortex continued for hours following a brief period of simultaneous sensory and nucleus basalis stimulation (Froemke et al., 2007). Additional sensory stimulation during postinduction periods such as these may lead to longer lasting changes.

It also appears that the influence of the additional stimulation spread into the subsequent periods of activation of the permissive signals, because learning was enhanced when the stimulus exposures preceded target-task performance. A potential explanation for the enhancement obtained with this presentation order is that additional stimulation increased the efficacy of the stimulation during periods of activation of the permissive signals. In this scenario, the additional stimulus exposures did not contribute to learning directly but rather increased the potential for improvement once the various requirements for malleability, such as the presence of the necessary permissive signals, were eventually met. This idea is reminiscent of demonstrations that the synaptic plasticity resulting from an inducing event can be altered by prior stimulation that does not produce plasticity itself (synaptic metaplasticity) (Abraham and Tate, 1997). It also evokes reports that even nonconscious exposure to a stimulus can subsequently lead to improved perception and identification of that stimulus (perceptual priming) (Wiggs and Martin, 1998), possibly due to a sharpening of the internal stimulus representation via a reduction in the responsiveness of neurons that are not crucial to the encoding of the key features of that stimulus (repetition suppression) (Desimone, 1996; Wiggs and Martin, 1998). Along this same line, there is recent evidence that electroencephalographic responses in the human central auditory system can be altered by sensory stimulation in the absence of task performance (Tremblay et al., 2010).

The suggestion that different processes may govern the enhancement obtained when the target-task performance preceded 
compared with when it followed the additional stimulus exposures receives some support from the tentative conclusion that the patterns of generalization differed for these two presentation orders. If identical processes were operating in the two orders, then the outcomes should not have differed between them. The implication that learning on a trained condition may lead to generalization to untrained conditions under some circumstances but not others is consistent with recent evidence that perceptual learning and its generalization may arise from changes that are at least partially distinct (Wright et al., 2010).

In this same framework, two other aspects of the temporal interaction between the permissive signals and additional stimulation are revealed by the characteristics of the additional stimulus exposures that did and did not yield enhancement. First, the circuitry supporting the interaction between these two elements seems to be selective for a particular stimulus feature rather than for the stimulus as a whole. Learning enhancement on frequency discrimination occurred here only when the additional stimulus exposures shared the same frequency with the standard stimuli used during target-task performance. Yet, the additional exposures did not have to be identical to those used during the target task to be effective. Second, the functional element of the required stimulation did not seem to arise from the physical presence of the signal variations to be discriminated. The additional stimulus exposures all had the same frequency, but nevertheless aided learning on frequency discrimination. This observation echoes a recent report that listeners improved on frequency discrimination following a single session of practice on that task using constantfrequency stimuli (Amitay et al., 2006), but extends this outcome to a multiple-day training regimen in which those stimuli were encountered while not performing the target task at all.

Finally, it is important to note that learning enhancement occurred even though the additional stimulus exposures were presented while the listeners were performing non-target tasks. The additional exposures thus were effective even though they were encountered during the generation of permissive signals that presumably were directed to different neural processes than were engaged by the target task. However, the observation that enhancement did not occur when the additional exposures had a different frequency than the standard in the target task diminishes the possibility that the mere presence of permissive signals is sufficient to generate learning enhancement.

On a practical level, the present results suggest a means by which perceptual training regimens might be made markedly more efficient and less effortful. The current data indicate that it may be possible to reduce the effort required by participants by at least half, with no deleterious effect, simply by combining periods of task performance with periods of additional stimulus exposure. If this proves to be a general rule of nondeclarative learning, it could help to explain how potent instances of learning can arise when sensory stimulation is not always coupled with attention (or some other permissive signal), including the acquisition of language (Kuhl, 2004) and the emergence of chronic pain (Flor and Diers, 2007).

\section{References}

Abraham WC, Tate WP (1997) Metaplasticity: a new vista across the field of synaptic plasticity. Prog Neurobiol 52:303-323.

Ahissar E, Vaadia E, Ahissar M, Bergman H, Arieli A, Abeles M (1992) Dependence of cortical plasticity on correlated activity of single neurons and on behavioral context. Science 257:1412-1415.

Ahissar M, Hochstein S (1993) Attentional control of early perceptual learning. Proc Natl Acad Sci U S A 90:5718-5722.
Ahissar M, Hochstein S (2004) The reverse hierarchy theory of visual perceptual learning. Trends Cogn Sci 8:457-464.

Amitay S, Irwin A, Moore DR (2006) Discrimination learning induced by training with identical stimuli. Nat Neurosci 9:1446-1448.

Banai K, Ortiz J, Oppenheimer JD, Wright BA (2010) Learning two things at once: Differential constraints on the acquisition and consolidation of perceptual learning. Neuroscience 165:436-444.

Bao S, Chang EF, Woods J, Merzenich MM (2004) Temporal plasticity in the primary auditory cortex induced by operant perceptual learning. Nat Neurosci 7:974-981.

Blake DT, Heiser MA, Caywood M, Merzenich MM (2006) Experiencedependent adult cortical plasticity requires cognitive association between sensation and reward. Neuron 52:371-381.

Clapp WC, Kirk IJ, Hamm JP, Shepherd D, Teyler TJ (2005) Induction of LTP in the human auditory cortex by sensory stimulation. Eur J Neurosci 22:1135-1140.

Cohen J (1988) Statistical power analysis for the behavioral sciences. In: The concepts of power analysis. Hillsdale, NJ: Erlbaum.

Crist RE, Kapadia MK, Westheimer G, Gilbert CD (1997) Perceptual learning of spatial localization: specificity for orientation, position, and context. J Neurophysiol 78:2889-2894.

Crist RE, Li W, Gilbert CD (2001) Learning to see: experience and attention in primary visual cortex. Nat Neurosci 4:519-525.

Desimone R (1996) Neural mechanisms for visual memory and their role in attention. Proc Natl Acad Sci U S A 93:13494-13499.

Fahle M (1997) Specificity of learning curvature, orientation, and vernier discriminations. Vision Res 37:1885-1895.

Flor H, Diers M (2007) Limitations of pharmacotherapy: behavioral approaches to chronic pain. Handb Exp Pharmacol:415-427.

Fritz J, Shamma S, Elhilali M, Klein D (2003) Rapid task-related plasticity of spectrotemporal receptive fields in primary auditory cortex. Nat Neurosci 6:1216-1223

Froemke RC, Merzenich MM, Schreiner CE (2007) A synaptic memory trace for cortical receptive field plasticity. Nature 450:425-429.

Gao E, Suga N (1998) Experience-dependent corticofugal adjustment of midbrain frequency map in bat auditory system. Proc Natl Acad Sci U S A 95:12663-12670.

Gilbert CD, Sigman M (2007) Brain states: top-down influences in sensory processing. Neuron 54:677-696.

Godde B, Stauffenberg B, Spengler F, Dinse HR (2000) Tactile coactivationinduced changes in spatial discrimination performance. J Neurosci 20:1597-1604.

Karni A, Sagi D (1991) Where practice makes perfect in texture discrimination: evidence for primary visual cortex plasticity. Proc Natl Acad Sci U S A 88:4966-4970.

Kuhl PK (2004) Early language acquisition: cracking the speech code. Nat Rev Neurosci 5:831-843.

Levi DM, Polat U (1996) Neural plasticity in adults with amblyopia. Proc Natl Acad Sci U S A 93:6830-6834.

Levitt H (1971) Transformed up-down methods in psychoacoustics. J Acoust Soc Am 49:467-477

Li W, Piëch V, Gilbert CD (2004) Perceptual learning and top-down influences in primary visual cortex. Nat Neurosci 7:651-657.

Maye J, Werker JF, Gerken L (2002) Infant sensitivity to distributional information can affect phonetic discrimination. Cognition 82:B101-B111.

Meinhardt G (2002) Learning to discriminate simple sinusoidal gratings is task specific. Psychol Res 66:143-156.

Meinhardt G, Grabbe Y (2002) Attentional control in learning to discriminate bars and gratings. Exp Brain Res 142:539-550.

Näätänen R, Schröger E, Karakas S, Tervaniemi M, Paavilainen P (1993) Development of a memory trace for a complex sound in the human brain. Neuroreport 4:503-506.

Nissen MJ, Bulemer P (1987) Attentional requirements of learning: evidence from performance measures. Cogn Psychol 19:1-32.

Polley DB, Steinberg EE, Merzenich MM (2006) Perceptual learning directs auditory cortical map reorganization through top-down influences. J Neurosci 26:4970-4982.

Recanzone GH, Merzenich MM, Jenkins WM, Grajski KA, Dinse HR (1992) Topographic reorganization of the hand representation in cortical area $3 \mathrm{~b}$ owl monkeys trained in a frequency-discrimination task. J Neurophysiol 67:1031-1056.

Recanzone GH, Schreiner CE, Merzenich MM (1993) Plasticity in the fre- 
quency representation of primary auditory cortex following discrimination training in adult owl monkeys. J Neurosci 13:87-103.

Saffran JR, Aslin RN, Newport EL (1996) Statistical learning by 8-monthold infants. Science 274:1926-1928.

Seitz A, Watanabe T (2005) A unified model for perceptual learning. Trends Cogn Sci 9:329-334.

Seitz AR, Dinse HR (2007) A common framework for perceptual learning. Curr Opin Neurobiol 17:148-153.

Shiu LP, Pashler H (1992) Improvement in line orientation discrimination is retinally local but dependent on cognitive set. Percept Psychophys 52:582-588.

Sigman M, Gilbert CD (2000) Learning to find a shape. Nat Neurosci 3:264-269.

Stefan K, Wycislo M, Classen J (2004) Modulation of associative human motor cortical plasticity by attention. J Neurophysiol 92:66-72.

Treisman A, Vieira A, Hayes A (1992) Automaticity and preattentive processing. Am J Psychol 105:341-362.
Tremblay KL, Inoue K, McClannahan K, Ross B (2010) Repeated stimulus exposure alters the way sound is encoded in the human brain. PLoS One 5:e10283.

Watanabe T, Náñez JE, Sasaki Y (2001) Perceptual learning without perception. Nature 413:844-848.

Weinberger NM (2004) Specific long-term memory traces in primary auditory cortex. Nat Rev Neurosci 5:279-290.

Wiggs CL, Martin A (1998) Properties and mechanisms of perceptual priming. Curr Opin Neurobiol 8:227-233.

Wright BA, Sabin AT (2007) Perceptual learning: how much daily training is enough? Exp Brain Res 180:727-736.

Wright BA, Zhang Y (2009) Insights into human auditory processing gained from perceptual learning. In: The cognitive neurosciences IV (Gazzaniga MS, ed), pp 353-366. Cambridge, MA: MIT.

Wright BA, Wilson RM, Sabin AT (2010) Generalization lags behind learning on an auditory perceptual task. J Neurosci 30:11635-11639. 